\title{
Modeling, Estimation, and Optimal Control of Anti-COVID-19 Multi-dose Vaccine Administration
}

This paper was downloaded from TechRxiv (https://www.techrxiv.org).

\section{LICENSE}

CC BY-NC-SA 4.0

SUBMISSION DATE / POSTED DATE

$12-10-2021 / 14-10-2021$

\section{CITATION}

Scarabaggio, Paolo; Carli, Raffaele; Cavone, Graziana; Epicoco, Nicola; Dotoli, Mariagrazia (2021): Modeling, Estimation, and Optimal Control of Anti-COVID-19 Multi-dose Vaccine Administration. TechRxiv. Preprint. https://doi.org/10.36227/techrxiv.16794724.v1

$\mathrm{DOI}$ 


\title{
Modeling, Estimation, and Optimal Control of Anti-COVID-19 Multi-dose Vaccine Administration
}

\author{
Paolo Scarabaggio, Graduate Student Member, IEEE, Raffaele Carli, Member, IEEE, \\ Graziana Cavone, Member, IEEE, Nicola Epicoco, and Mariagrazia Dotoli, Senior Member, IEEE
}

\begin{abstract}
The recent trends of the COVID-19 research are being devoted to disease transmission modeling in presence of vaccinated individuals, while the emerging needs are being focused on developing effective strategies for the optimal distribution of vaccine between population. In this context, we propose a novel non-linear time-varying model that effectively supports policy-makers in predicting and analyzing the dynamics of COVID-19 when partially and fully immune individuals are included in the population. Specifically, this paper proposes an accurate SIRUCQHE epidemiological model, with eight compartments (namely, Susceptible, Infected, Removed, Unsusceptible, Contagious, Quarantined, Hospitalized, and Extinct). Differently from the related literature, where the common strategies typically rely on the prioritization of the different classes of individuals, we propose a novel Model Predictive Control approach to optimally control the multi-dose vaccine administration in the case the available number of doses is not sufficient to cover the whole population. Focusing on the minimization of the expected number of deaths, the approach discriminates between the number of first and second doses, thus considering also the possibility that some individuals may receive only one injection if the resulting expected fatalities are low. To show the effectiveness of the resulting strategies, we first calibrate the model on the Israeli scenario using real data to get reliable predictions on the pandemic dynamics. Lastly, we estimate the impact of the vaccine administration on the virus dynamics and, in particular, based on validated model, we assess the impact of the first dose of the Pfitzer's vaccine confirming the results of clinical tests.
\end{abstract}

COVID-19, pandemic modeling, vaccine, vaccine distribution, model predictive control.

\section{INTRODUCTION}

In order to properly analyze the spread of COVID-19 contagions, predict its dynamics, and support decision makers in defining effective mitigating actions, several mathematical models have been developed. All these models are variants of the classical SIR-based compartmental epidemiological model, which considers the classes of Susceptible, Infective, and Recovered individuals. Other compartments are typically added to deepen the analysis (see, for instance, the review in [1]).

One of the main problems in epidemiological modelling relies in the proper estimation of the involved parameters, re-

The work of M. Dotoli is partially supported by Italian University and Research Ministry under project RAFAEL (National Research Program, contract n. ARS01-00305).

P. Scarabaggio, R. Carli, G. Cavone, and M. Dotoli are with the Department of Electrical and Information Engineering of the Polytechnic of Bari, Italy. (e-mail: \{paolo.scarabaggio, raffaele.carli, graziana.cavone, mariagrazia.dotoli\}@poliba.it).

N. Epicoco is with the Department of Information Engineering, Computer Science and Mathematics (DISIM) and with the Center of Excellence DEWS (Design methodologies for Embedded controllers, Wireless interconnect and System-on-chip), University of L'Aquila, L'Aquila, Italy (e-mail: nicola.epicoco@univaq.it). quiring widespread data, which are however seldom available in the early stages of any pandemic. With the ongoing of the disease, the increased amount of available data has lead to more detailed models, where much more compartments have been in turn considered to properly represent the COVID-19 dynamics. For instance, a SIDARTHE model is presented in [2], where the population is divided into Susceptible, Infected, Diagnosed, Ailing and Recognized, Threatened, Healed, and Extinct. Similarly, the model presented in [3] divides the population into Susceptible, Exposed, symptomatically Infected, Asymptomatically infected, Hospitalized, and Recovered compartments.

In addition, since many countries worldwide are organized into separate administrative areas with different shares of the national healthcare system, another group of works deepens the analysis at a regional level, thus allowing to take into account the economic and social differences existing in each single area of a country. As an example of contributions in this field, the work in [4] proposes a six compartments model (dividing the regional population in Susceptibles, Infected, Hospitalized, Quarantined, Deceased, and Recovered), and our paper in [5] presents a multi-region SIRQTHE model (composed by the classes of Susceptible, Infected, Removed, Quarantined, Threatened, Healed, and Extinct individuals.

The first COVID-19 vaccines have already begun to be administered to population in all countries. Accordingly, the recent trends of the COVID-19 research is directed towards the proper choice of the type of vaccine, its distribution, and dosage form, which can directly impact the immune responses induced and the resultant efficacy against the COVID-19 outbreak [6]. Consequently, most contributions on COVID19 modeling are focusing on models that include additional classes of the population to suitably represent vaccinated individuals [7]-[10]. At the same time, several countries around the world are struggling to complete the vaccination campaigns in the shortest time possible. However, the limited amount of vaccine doses is making this task challenging and almost impossible in a short period. In fact, it will probably take time before there is enough vaccine for all the population, thus evoking the question on how to distribute it in the most effective manner [11].

As for other epidemics, intelligent decision making strategies have been effectively used to plan the vaccination in the absence of a sufficient number of doses. For the interested readers, the authors in [12] review the scientific literature on mathematical models and on optimal control theory to compute the optimal strategies aimed at reducing the spread of an infectious disease. 
As for COVID-19, several vaccine allocation strategies have been proposed [13]. For instance, in [14] the authors employ a mathematical model to compare five age-stratified prioritization strategies in order to reduce existing inequities in COVID-19 vaccine distribution. In [15] a model for the optimal vaccine allocation is proposed and applied for the Korean scenario. The approach aims at minimizing infections, deaths, and years of life lost while accounting for population factors, such as country-specific age distribution and contact structure, and various levels of vaccine efficacy. The strategic spatio-temporal distribution of vaccines is investigated in [11], with the final aim of prioritizing the distribution in those areas where there are the most new cases of infection during a fixed time period and compare it with the common practice of distributing vaccines demographically. Referring to optimal control strategy, in $[8]$ the authors propose an optimal control strategy for vaccine administration in the COVID-19 pandemic. The work considers both the minimization of the infected individuals and the prescribed vaccine concentration during the treatment.

A viable option proposed by some researchers to overcome the lack of vaccine for the COVID-19 pandemic lies in delaying the injection of the second dose. To date, the UK is the only country that is delaying the second dose to 12 weeks [16]. Indeed, some researches point out that this unproven dosing schedule introduced without fully informed patient consent may bring several risks for individuals and the population. Clinical trials suggest that delaying the second dose to 12 weeks may lower the efficacy of the vaccine until a point where the effect of the second dose becomes negligible and thus comparable with a single-dose vaccination. In fact, clinical reports demonstrate $52,4 \%$ of efficacy after 22 days for BNT162b2 Pfizer's vaccine first dose [17]. Moreover, an effectiveness of 50-60\% in tackling the COVID-19 infections has been reported in observational cohort studies in Israel covering the same period [18].

Therefore, the high efficacy with a single dose may be sufficient for the herd immunity in absence of a sufficient number of vaccine; not surprisingly, the World Health Organization defined as a minimum $50 \%$ threshold for a vaccine to be effective, and thus a single dose can still be useful to reduce the pandemic spread when no other options are available [19].

In addition this paper we revise and extend our previous contribution in [5] by presenting a novel time-varying SIR-based epidemiological model that better fits the current epidemiological situation in the presence of vaccination. In detail, we modify propose a SIRUCQHE epidemic model, with eight compartments (namely, Susceptible, Infected, Removed, Unsusceptible, Contagious, Quarantined, Hospitalized, and Extinct).

Differently from our previous contribution [5], we divide Infected individuals into Contagious and Infected yet not contagious, and we assume that Threatened people must observe a quarantine period when released from the hospital. Moreover, we remove the time dependency of the recovery rate and we substitute the time dependency of the death rate with a function dependent on the number of Threatened people.

We deem it essential to remark that, while other literature contributions dynamically identify the model parameters by dividing the fitting period, in our work time-varying functions are presented to model the time-varying parameters. Furthermore, since the infection rate depends on people's mobility, differently from the related literature, in the fitting phase we make use of the Google mobility reports [20] to identify and predict the evolution of the infection rate. At the same time, we use the ratio between new daily discovered cases and the daily swabs to consider the variability of the so-called detection rate.

In addition, with respect to our previous contribution [5] where the control actions are focused on enforcing restrictions on people mobility, in this work we also include in the model vaccinations and an additional class to represent partially immune individuals, i.e., people that received only a single vaccine dose. Moreover, we apply the aforementioned model in conjunction with a Model Predictive Control (MPC) approach aiming at optimally planning the vaccine distribution minimizing the number of fatalities. Differently from the related literature, where the control actions aim at optimally distributing the vaccine among individuals, in the proposed approach, we assume that, in absence of a sufficient number of vaccines, decision makers can decide to employ a single vaccine dose.

To date, Israel has administered the most COVID-19 vaccine doses per capita in the world. More than 3.67 million Israelis have received the first dose of the BioNTech-Pfizer mRNA vaccine since the vaccination campaign began on December 20. This represents about $40 \%$ of the country's population. More than $28 \%$ have already received the second dose [21].

Therefore, we apply the proposed approach to the Israeli scenario firstly estimating the effectiveness of a single vaccine dose to tackle the COVID-19 among a population and secondly showing the reliability of the proposed control model in selecting the best strategy while minimizing the numbers of fatalities.

The rest of this work is structured as follows. Section $\amalg$ presents the SIRUCQTHE model, its dynamical equations, and the parameters identification process. Section III proposes the MPC framework, describing the corresponding control variables, objectives, and constraints, and formulating the comprehensive optimal allocation problem. Section IV reports the numerical outcomes achieved by the simulations based on the real data of the Israeli scenario. Finally, Section V concludes the paper highlighting possible future developments.

\section{COVID-19 Dynamics with Multi-Dose VACCINATION}

Typically, compartmental models are considered appropriate to model the proliferation of a virus within a large population. In these models, the population is partitioned into compartments and can flow from one compartment to another according to specific flow rates. The most traditional compartmental models are the SIR-based models, for which it can be assumed that the pandemic dynamics is faster than the dynamics of births and deaths.

In this paper, a novel time-varying discrete-time epidemiological model for the COVID-19 spread in presence of 


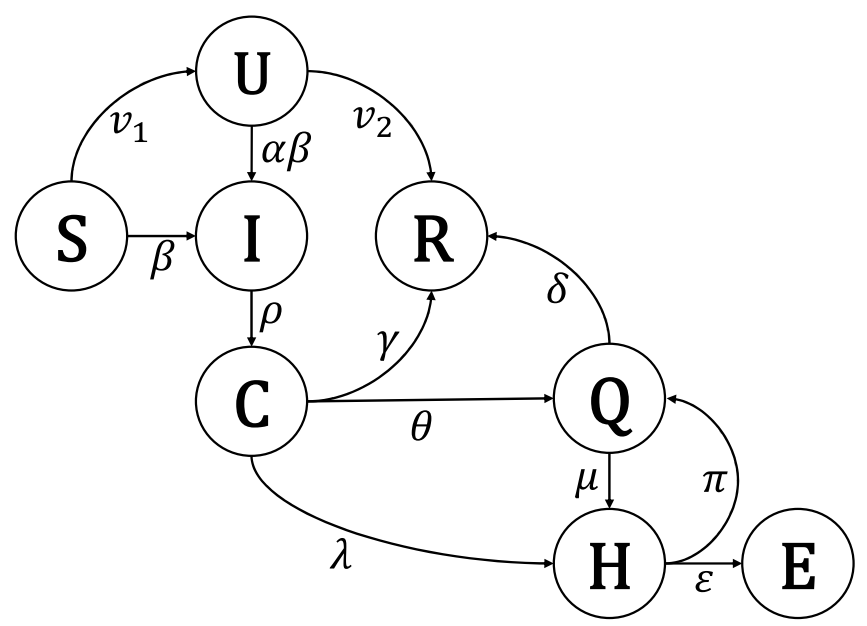

Fig. 1. Scheme of the SIRUCQHE model.

partially and fully vaccinated individuals is proposed, named SIRUCQHE, which classifies individuals into eight classes. More in detail, the overall population is divided into the following compartments:

- Susceptible;

- Infected (infected by someone and not yet contagious);

- Removed (fully vaccinated or completely recovered);

- Unsusceptible (vaccinated and partially immune);

- Contagious (infected and undetected, contagious);

- Quarantined (infected and detected)

- Hospitalized (hospitalized in life-threatening or noncritical situation);

- Extinct (detected and dead).

The SIRUCQHE model is thus composed of eight timevarying difference equations, which represent the dynamics of individuals' flows between the various compartments. We consider as state variables of the model the fraction of the overall population related to the various compartments. Denoting by a capital Latin letter each state variable, and denoting the time step as $k$, the model can be written as follows:

$$
\begin{aligned}
& S(k+1)=S(k)-\beta(k) \frac{C(k) S(k)}{N}-v_{1}\left(k-\tau_{1}\right) \\
& I(k+1)=I(k)+\beta(k) \frac{C(k) S(k)}{N}+\alpha \beta(k) \frac{C(k) U(k)}{N}-\rho I(k) \\
& R(k+1)=R(k)+\gamma C(k)+\delta Q(k)+v_{2}\left(k-\tau_{2}\right) \\
& U(k+1)=U(k)-\alpha \beta(k) \frac{C(k) U(k)}{N}+v_{1}\left(k-\tau_{1}\right)-v_{2}\left(k-\tau_{2}\right) \\
& C(k+1)=C(k)+\rho I(k)-(\gamma+\theta(k)+\lambda) C(k) \\
& Q(k+1)=Q(k)+\theta(k) C(k)+\pi H(k)-(\delta+\mu) Q(k) \\
& H(k+1)=H(k)+\mu Q(k)+\lambda C(k)-(\pi+\varepsilon(k)) H(k) \\
& E(k+1)=E(k)+\varepsilon(k) H(k)
\end{aligned}
$$

where $N$ represents the whole population.

The eight classes are connected by directed arcs whose weights correspond to the parameters that put in relation the corresponding classes. The overall interconnections between the above compartments are shown in Fig.1.

In particular, $\beta(k) \in \mathbb{R}_{+}$is the time-varying infection rate, whose value depends on the population behavior and the adopted social distancing measures. The parameter $\alpha \in[0-1]$ describes how effective a single dose of the vaccine is, e.g. when $\alpha=0$ only the first injection is fully effective to prevent an infection. Moreover, $v_{1}(k) \in \mathbb{Z}_{+}$and $v_{2}(k) \in \mathbb{Z}_{+}$represent the number of first and second vaccine doses made at time $k$, respectively. Parameters $\tau_{1} \in \mathbb{Z}_{+}$and $\tau_{2} \in \mathbb{Z}_{+}$define the average time required to develop an immune response after the first and the second dose. Parameter $\theta(k) \in \mathbb{R}_{+}$is the timevarying detection rate that describes the rate of Contagious people that are recognized and Quarantined; this must be modeled as a time-varying parameter since it largely depends on the epidemiological situation. As will be demonstrated in Section IV parameter $\beta(k)$ can be correlated with people's mobility through the use of the Google Mobility Reports [20] and parameter $\theta(k)$ with the ratio between new daily discovered cases and daily swabs. Parameter $\rho \in \mathbb{R}_{+}$is the so-called incubation rate. Parameters $\gamma \in \mathbb{R}_{+}$and $\delta \in \mathbb{R}_{+}$are the so-called healing rates. However, the first describes the rate of healing of Contagious and unrecognized people (thus, not requiring hospitalization) that are no longer infectious. In contrast, the second is the healing rate of Quarantined people who can leave this class when they are no longer infectious or legally obliged to stay at home. Parameters $\lambda \in \mathbb{R}_{+}$and $\mu \in \mathbb{R}_{+}$are the hospitalization rates: $\lambda$ is the rate of people recognized and immediately hospitalized when a severe symptomatic condition occurs, while $\mu$ is the rate of Quarantined people that need for hospitalization. Parameter $\pi \in \mathbb{R}_{+}$is the healing rate of Threatened people. Note that we assume that, after being released from the hospital, people must observe a quarantine period. Lastly, $\varepsilon(k) \in \mathbb{R}_{+}$ is the time-varying death rate that depends on the number of Threatened people. This relation is discussed in Section IV

Note that, based on the current level of knowledge, we reasonably assume that the probability of becoming susceptible after being healed is negligible since the relative dynamics is much slower than the main time constant of the model [22].

The proposed model ensures a good accuracy, in representing the dynamics of the disease, also allowing to represent all the facets of the pandemic diffusion. Its simplicity helps identifying the characteristic parameters starting from the available data, thus motivating its application to real systems. In fact, various papers aiming at representing the dynamics of the COVID-19 pandemic present compartmental models, but generally lack an accurate identification of the model parameters. Therefore, with respect to the related literature in the SIRUCQHE model we compress or eliminate some classes, and we disregard some connections between compartments. Despite these simplifying assumptions, as shown in Section IV the presented model is effective in the identification phase by only requiring a minimal set of epidemiological data.

\section{Optimal Vaccine Multi-Dose Administration}

In this section we describe the proposed approach to optimally plan the multi-dose vaccine administration in order to reach the mass coverage and thus heard immunity; however, we assume that the approach takes into account the limited availability of vaccine.

We employ a receding horizon scheme and we assume that the prediction and control horizon have the same length. 
Specifically, at the generic sampling step $h \in \mathbb{Z}_{+}$the horizon - defined as $\mathcal{K}(h)=\{h, \ldots, h+K-1\}$ - contains $K$ equally spaced time slots with length $\Delta_{k}$.

Moreover, for each time step $h$ we define a vector of control variables $\mathbf{u}(h):=\left(\mathbf{u}_{1}(h)^{\top}, \mathbf{u}_{2}(h)^{\top}\right)^{\top} \in \mathbb{Z}_{+}^{2 K}$ where $\mathbf{u}_{1}(k):=\left(u_{1}(h), \ldots, u_{1}(h+K-1)\right)^{\top} \in \mathbb{Z}_{+}^{K}$ and $\mathbf{u}_{2}(k):=$ $\left(u_{2}(h), \ldots, u_{2}(h+K-1)\right)^{\top} \in \mathbb{Z}_{+}^{K}$ collect the quantities of first and second doses that are planned to be administered over the given control horizon, respectively.

In order to avoid too frequent and impractical changes in the strategies, the control actions are kept constant over a given period equal to $\Delta_{l}=\omega \Delta_{k}$ (i.e., for $\omega$ time slots). For instance, if $\Delta_{k}$ corresponds to one day, it could be meaningful to set the periodicity of the control actions equal to one week (i.e., $\omega=7$ ). Assuming that $K=L \omega$, with $L \in \mathbb{N}$, the following additional constraints are then introduced to keep the control actions constant in each period $l$ :

$$
\begin{gathered}
u_{1}(\omega l+1)=u_{1}(\omega l+2)=\cdots=u_{1}(\omega l+\omega), \forall l=0, \ldots, L-1 \\
u_{2}(\omega l+1)=u_{2}(\omega l+2)=\cdots=u_{2}(\omega l+\omega), \forall l=0, \ldots, L-1 .
\end{gathered}
$$

Furthermore, as we aim at planning the vaccine distribution in the case the number of doses are scarce, we include an additional constraint on the total number of available doses. Since in most countries the vaccine doses are not distributed on a daily basis, we assume this constraint must be fulfilled over the given period equal to $\Delta_{l}$. As a consequence, let us define the aforementioned constraint as:

$$
\sum_{i=1}^{\omega} u_{1}(\omega l+i)+u_{2}(\omega l+i) \leq \bar{v}(l+1), \forall l=0, \ldots, L-1
$$

where vector $\bar{v}(l+1)$ is the maximum number of vaccine doses for the period $l$.

As the number of people in a compartmental model cannot be negative, we include the following constraints that should be respected while planning the vaccine administration:

$$
\begin{gathered}
v_{1}(k) \leq S(k) \\
v_{2}(k) \leq U(k) .
\end{gathered}
$$

Lastly, we assume that the number of second doses cannot be chosen independently from the previously made first doses. In fact, clinical tests reported a maximum efficacy for the second dose only if this is administered no later than a given maximum time period from the administration of the first dose. Therefore, here we assume that the second dose can be made only between a characteristic time window after the first injection. Moreover, we assume that if a person does not receive the second injection in this time period then it will not receive it anymore. The resulting constraint can be written as:

$$
\begin{aligned}
& v_{2}(k) \leq \sum_{\tau_{1}=\tau_{1}^{\min }}^{\tau_{1}^{\max }} v_{1}\left(k-\tau_{1}\right)-\cdots \\
& \cdots-\min \left\{0, v_{2}(k-1)-\sum_{\tau_{1}=\tau_{1}^{\min }+2}^{\tau_{1}^{\max }+1} v_{1}\left(k-\tau_{1}\right)\right\}
\end{aligned}
$$

where $\tau_{1}^{\min }$ is the minimum time that must elapse between the first and the second dose (e.g, for the BNT162b2 Pfizer's vaccine this is 21 days) and $\tau_{1}^{\max }$ is the maximum delay that can be accepted for the second injection.

Having defined the constraints that the vaccine distribution must follow, let us remark that the proposed MPC approach aims at optimizing the distribution of the vaccine doses while minimizing the number of deaths. Therefore, on the basis of the above defined control variables and related constraint set and the corresponding state model, the optimal control problem is defined as follows:

$$
\begin{array}{cl}
\underset{\mathbf{u}(h)}{\operatorname{minimize}} & J(\mathbf{u}(h))=E(h+K-1)-E(h) \\
\text { subject to } & S I R U C Q H E \text { model (1)-(8), } \forall k \in \mathcal{K}(h) \\
& \text { constraints } 9 \text {-14, } \forall k \in \mathcal{K}(h) .
\end{array}
$$

For the sake of taking the variability and uncertainty of the model parameters into account, we employ a rolling horizon framework; hence, we solve the optimization problem (15) at each $\omega$ time step in an iterative manner, based on the most recent input data. The obtained control actions related to the first time step are applied to the system to steer its behavior to the desired one, while the horizon is shifted forward. Then, a new optimization problem is solved over the shifted prediction horizon, based on the updated information on forecasts and system states.

It must be noticed that the presented closed-loop feedback control technique may depend on quantities that are both directly and not directly measurable. In effect, an identification procedure should be performed at each time step for the SIRUCQHE model, since the related classes cannot be not all directly estimable. In particular, the identification procedure consists in using, at each time step, the most recent data related to the available classes to dynamically update the remaining SIRUCQHE parameters. We discuss this aspect in the case study section.

\section{CAse STUdy}

This section is divided into two parts. In the first part, we describe the main assumptions on the system parameters and the fitting procedure, while in the second part we present some numerical simulations based on the proposed predictive control framework. The published and updated version of this experimental section can be found in [23].

\section{A. Parameters Identification}

Several epidemiological models have been proposed in the literature for modeling the dynamics of COVID-19. Let us explain how we calibrate the SIRUCQHE model with respect to the Israeli scenario based on real data obtained from [24].

The main problem related to COVID-19 epidemiological data available worldwide is that they represent a screenshot of the epidemiological situation each day. For each class, it is only available the number of people per each day without any indication about the flows within the different categories. This problem makes it hard to estimate the model parameters requiring additional assumptions. Hereafter, we detail the assumptions considered for each of the model parameters. 
1) $\beta(k)$ : The so-called infection rate $\beta(k)$ for the COVID19 pandemic is usually assumed within 0.25 and 0.8 in the absence of any social distancing policies and people awareness. Following [25], [26] and [27], we assume that the infection rate is strongly influenced by people's mobility in different socioeconomic categories denoted by $\mathcal{G}=\{1, \ldots, G\}$. Hence, we assume that the infection rate can be decomposed into different terms each of which is mainly affected by a given category. Given the results obtained in several preliminary experiments on real data, we consider that a reduction of the mobility associated with the different socio-economic categories can be, with a good approximation, represented with a linear decrease of the infection rate as follows:

$$
\beta(k)=\beta_{0}+\boldsymbol{\beta}^{\top} \mathbf{m}(k)
$$

where $\beta_{0}$ is the infection rate not explicable with a variation of the mobility (e.g., household infection), $\boldsymbol{\beta}=\left(\beta_{1}, \ldots, \beta_{G}\right)^{\top}$ is the column vector collecting the term $\beta_{g}$ of the infection rate corresponding to each socio-economic category $g \in \mathcal{G}$, and $\mathbf{m}(k)=\left(m_{1}(k), \ldots, m_{G}(k)\right)^{\top}$ is the column vector collecting the mobility level $m_{g}(k)$ at time step $k$ for each socioeconomic category $g \in \mathcal{G}$. We assume that $m_{g}(k)=1$ when the $g$-th category's mobility level equals the nominal value, i.e., the value in absence of mobility restrictions.

We estimated the evolution of people's mobility in different categories through the Google Mobility Reports [20]. These reports show how visits and length of stay at different places change compared to a baseline. We select the Workplaces, Retail \& recreation, and Public transport categories as the most significant in terms of socio-economic importance. Hence, we define $\mathbf{m}(k)=\left(m_{1}(k), m_{2}(k), m_{3}(k)\right)^{\top}$ and $\boldsymbol{\beta}=\left(\beta_{1}, \beta_{2}, \beta_{3}\right)^{\top}$, indicating respectively the mobility level of Workplaces, Retail and recreation and Public transport.

2) $\rho$ : The so-called incubation rate $\rho$ is the rate of infected people that become contagious and can consequently infect other people. In the literature, the incubation time is estimated between 2 and 7 days $[28]-[30]$.

3) $\gamma$ : The healing rate of unrecognized Contagious people $\gamma$ can be approximated by a constant. In particular, literature findings show that the healing time is approximately 14 days. However, in our model, we do not remove people from the Contagious compartment when they are completely healed, but only when they are not contagious or have a really low viral load. In the literature, this period is estimated between 3 and 10 days [31]-[34].

4) $\delta$ : The healing rate of Quarantined people who do not need hospitalization $\delta$ can also be approximated by a constant value and can be substituted with $\gamma$ when a person is removed from the Quarantined compartment immediately after he/she becomes not contagious. However, in several countries, someone may be forced to be in quarantine even after being clinically healed because the procedure requires two negative tests. In several countries the quarantine period cannot be shorter than 10-14 days, depending on the swabs' tests results.

5) $\theta(k)$ : Parameter $\theta(k)$ models the rate of Contagious people recognized and Quarantined, the so-called detection rate. This is mainly related to the specific policy adopted by each region and the number of laboratory testing capacities (e.g., in terms of tested swabs). Numerous research studies pointed out that the ratio $r(k)=p(k) / w(k)$ between new daily discovered cases $p(k)$ and the daily swabs $w(k)$ is crucial to understand how the tracking system is operating. When this ratio increases, it means that the tracking system is not working well and that the pandemic is out of control, i.e., the real number of infected people is much higher than the recognized cases. Conversely, when this number is low, it means that few cases have been recognized with respect to the total amount of analyzed swabs: therefore, the pandemic is under control. Note that the aforementioned assumptions only hold when the tests are made in a stochastic way. In fact, if a country has a well-organized tracking system, it can perform only the necessary tests on people surely infected. Therefore, to consider the variability of parameter $\theta(k)$, we relate it with the aforementioned ratio as:

$$
\theta(k)=\theta_{0}(1-r(k)) .
$$

It should be noted that this parameter is the most critical in COVID-19 modeling due to the high number of asymptomatic individuals. Therefore, this relation is not accurate but our experiments show that it helps the results of the identification process.

6) $\lambda$ and $\mu$ : These parameters are the hospitalization rates, i.e., the rate of people recognized only when severe symptomatic conditions occur and the rate of Quarantined people to be hospitalized, respectively. We assume these two parameters are constant.

7) $\pi$ : The recovery rate $\pi$ during the first outbreak of COVID-19 was far from being constant; in fact, the national healthcare system was not prepared and did not have healing procedures. However, after the implementation of new standardized clinical approaches, we can assume that this parameter is constant.

8) $\varepsilon(k)$ : The death rate $\varepsilon$ at the beginning of a new epidemic is not constant and hopefully decreases with time. This is mainly due to the availability of new clinical treatments. The death rate for each time step $k$ can be formulated as:

$$
\varepsilon(k)=(E(k)-E(k-1)) / T(k) .
$$

With the pandemic's development, this parameter becomes easily identifiable, and it only depends on how much the healthcare system is under pressure.

9) $\tau_{1}$ and $\tau_{2}$ : The delays $\tau_{1}$ and $\tau_{2}$ are the characteristic average times required to develop an immune response after the first and the second vaccine doses. These values vary depending on the vaccine.

In order to estimate the above listed parameters of the proposed SIRUCQHE model, a least-squares optimization technique is adopted combined with constraints to enforce the prior scientific knowledge of the COVID-19 pandemic. In detail, based on the aforementioned assumption and the main literature findings, we define specific bounds on each parameter summarized in Table 1

We firstly estimate the death rate, that we assume to follow a linear relation with the number of hospitalized individuals with coefficients $\varepsilon_{0}$ and $\varepsilon_{1}$. The estimation of such coefficients consists in minimizing the mean squared error (MSE) of the 
TABLE I

SIRUCQTHE MOdEl PARAMETER Bounds

\begin{tabular}{|c|c|c|c|c|}
\hline & Description & Compartments & Bounds & Reference \\
\hline$\beta_{0}$ & Base infection rate & $S \rightarrow I$ & {$[0.01,0.1]$} & $44,35,36$ \\
\hline$\beta_{g}$ & Mobility coefficients & $S \rightarrow I$ & {$[0.1,0.5]$} & $4,35,50$ \\
\hline$\rho$ & Incubation rate & $I \rightarrow C$ & {$[0.15,0.5]$} & [28]-50] \\
\hline$\theta_{0}$ & Detection rate & $C \rightarrow Q$ & {$[0.001,0.5]$} & 4,36 \\
\hline$\gamma$ & Healing rate & $C \rightarrow R$ & {$[0.1,0.3]$} & 4 , 32, [33], 37] \\
\hline$\delta$ & Healing rate & $Q \rightarrow H$ & {$[0.01,0.1]$} & 4,36 \\
\hline$\lambda$ & Threatening rate & $C \rightarrow T$ & {$[0.001,0.02]$} & $\sqrt{2}, \sqrt{4}$ \\
\hline$\mu$ & Threatening rate & $Q \rightarrow T$ & {$[0.001,0.08]$} & $\sqrt{2}, \sqrt{4}$ \\
\hline$\pi$ & Healing rate & $T \rightarrow H$ & {$[0.01,0.2]$} & {$[2,[4],[36], 38]$} \\
\hline$\tau_{1}$ & Approx. delay & $S \rightarrow U$ & 21 days & 17 \\
\hline$\tau_{2}$ & Approx. delay & $U \rightarrow R$ & 7 days & 17 \\
\hline
\end{tabular}

linear approximation with respect to the real data, which is defined as:

$$
\operatorname{MSE}\left(\varepsilon_{0}, \varepsilon_{1}\right)=\frac{1}{K} \sum_{k=1}^{K}\left(\frac{\left(\varepsilon_{0}+\varepsilon_{1} T(k)\right)-\varepsilon(k)}{\varepsilon(k)}\right)^{2}
$$

Consequently, by defining $\Xi_{1}$ as the vector collecting all the unknown parameters and the initial conditions are the only parameters to be estimated, we minimize the MSE of the model with respect to real data as follows:

$$
\begin{aligned}
\operatorname{MSE}\left(\Xi_{1}\right)= & \frac{1}{K} \sum_{k=1}^{K}\left(\frac{\hat{Q}\left(\Xi_{2}, k\right)-Q(k)}{Q(k)}\right)^{2} \\
& +\frac{1}{K} \sum_{k=1}^{K}\left(\frac{\hat{H}\left(\Xi_{2}, k\right)-H(k)}{H(k)}\right)^{2}
\end{aligned}
$$

Moreover, based on serological testing data [39], when searching for realistic parameters we assume that $C(k)<$ $20(Q(k)+H(k))$. For the sake of brevity we do not report here the results of the comparison between simulation model and the actual data of the considered case study. However, we report that the maximum error for the considered scenario on the Hospitalized class is approximately $20 \%$.

In order to estimate the impact of the vaccination on the model parameters, the identification is divided into two different phases. In the first phase, we estimate the model parameters in absence of vaccination, i.e., before the Israeli vaccination campaign started on December 19, 2020. In this way, we define reasonable and tight bounds for the parameters. Consequently, in the second phase, we perform the identification with the epidemiological data during the vaccination campaign to estimate the parameter $\alpha$ to assess the effectiveness of a single vaccine dose. We highlight that, in this phase, we use the previously defined bounds on the remaining parameters to make an accurate estimation of the vaccination impact. The results show that this parameter is approximately halving the infection rate, i.e., $\alpha=0.5851$, thus confirming the results of the clinical reports.

In Fig. 2, we show the results of the SIRUCQHE for compartments $H$ and $E$. In particular, we show the real data (with the effect of the vaccination campaign) and the forecasting of the model not considering the vaccinations. From the figure, it is clear the impact that the vaccination campaign had on preventing another outbreak of the virus. (a)

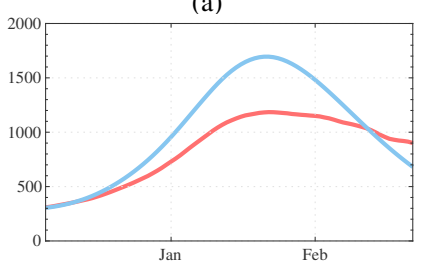

Fig. 2. Real data (red line) and results of the simulation (blue line) for the SIRUCQHE model without the impact of the vaccination campaign: Hospitalized (a) and Extinct (b).

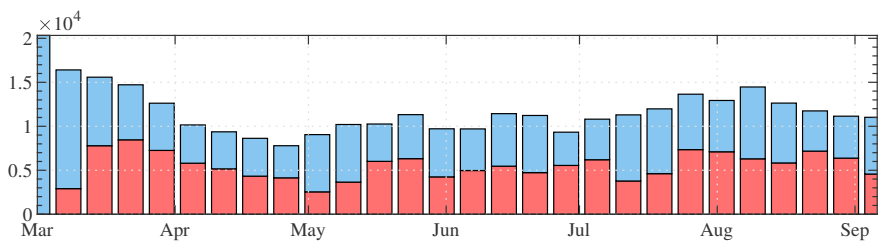

Fig. 3. Vaccine doses distribution over the simulation horizon: first doses (blue) and second doses (red).

\section{B. Optimal Distribution of the Vaccine Doses}

This subsection describes the numerical simulations based on the proposed MPC approach aimed at optimally controlling the doses' distribution. We test the approach over a simulation period of 20 weeks starting from March 5, 2021, using a prediction horizon of 10 weeks. The sampling interval $\Delta_{k}$ is set to one day. A daily change of the vaccination measures would be unrealistic in a real-life scenario, therefore, we assume weekly based control actions (i.e., $L=6$ and $\Delta_{l}=\omega \Delta_{k}$, with $\omega=7$ ). We consider the availability of a variable quantity of the vaccine to simulate shortage in the distribution. As for time parameters $\tau_{1}^{\min }$ and $\tau_{1}^{\max }$, we select them based on the BNT162b2 Pfizer's vaccine clinical report and thus we set them equal to 21 and 35 days, respectively.

The optimal control problem is implemented in MATLAB R2020a [40] using the Global Optimization toolbox on a laptop with a $1.3 \mathrm{GHz}$ Intel Core i7 CPU and 8 GB RAM.

Note that at each iteration of the MPC approach the above described identification procedure must be performed, since the characteristic parameters may vary during the pandemic spread.

As a first result, in Fig. 3 we show the administration of the first and the second dose. In particular, we show a stacked bar chart that indicates in blue the administration of the first doses of vaccine and in red the second one. The proposed control strategy is able to dynamically modify the vaccination plan even with a sudden change in the vaccine administration while minimizing the number of fatalities.

Furthermore, we show the effectiveness of the proposed procedure in Fig. 4, where we plot the results of the numerical simulation for compartments $S, R, C, U, H$, and $E$ under the effects of the MPC control. First of all we note that the dynamics of the Contagious and Hospitalized compartments (Fig. 4 (c) and Fig. 4 (e), respectively) tends to zero in about three months, conversely, after the same amount of months the number of Unsusceptible and Extinct people (Fig. 4 (d) 
(a)

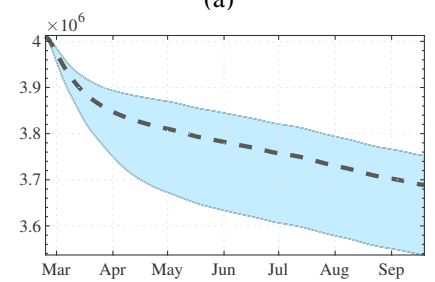

(c)

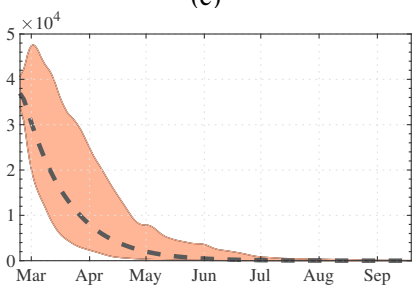

(e)

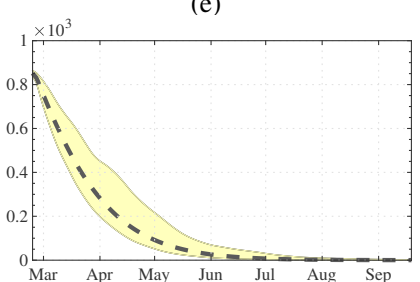

(b)

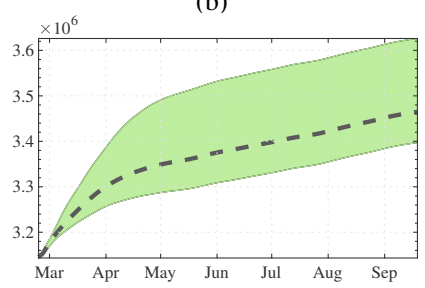

(d)

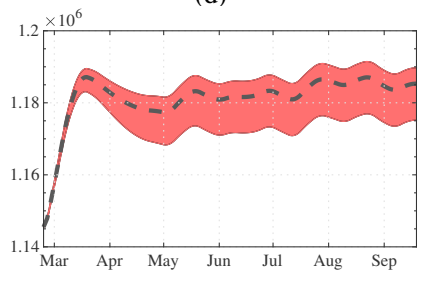

(f)

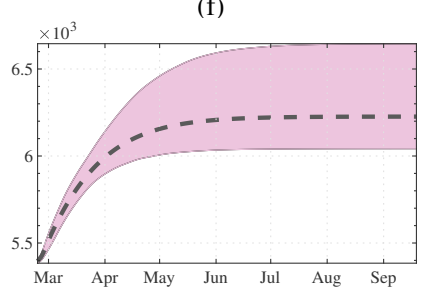

Fig. 4. Results of the simulation for the SIRUCQHE model: Susceptible (a), Removed (b), Contagious (c), Unsusceptible (d), Hospitalized (e), Extinct (f). Results of the simulation (dashed line) and the $20 \%$ estimated bounds (colored bands).

and Fig. 4(f), respectively) tend to steady state. In the figure, we add also an estimation of the system dynamics made by modifying the characterized parameters by $20 \%$ indicated by the colored bands. The results show that the proposed approach can effectively reduce the development of the pandemic leading to pandemic extinction after few months even in the presence of uncertainty. It is worth noting that, at the end of the simulation, almost 1.3 million people receive only a single injection. This confirms that, in order to reach herd immunity in the shortest time, it is possible to administrate a single dose of vaccine to the population.

\section{CONCLUSIONS}

Due to the limited amount of currently available vaccine doses, to date, it is crucial to develop suitable methods to support policy-makers in efficiently planning the vaccine distribution between population. To answer this challenging need, this paper presents a novel epidemiological SIR-based model aimed at representing the system dynamics and predicting its evolution when partially and fully immune individuals are included. More in detail, the epidemiological model consists in a novel non-linear time-varying SIRUCQHE model, so that eight different compartments of individuals and the connections between them are modeled to ensure a detailed representation of the pandemic's dynamics under vaccine administration: Susceptible, Infected, Removed, Unsusceptible, Contagious, Quarantined, Hospitalized, and Extinct. Leveraging on this model, and aiming at minimizing the expected number of

deaths, we present a novel Model Predictive Control approach to optimally allocate the number of first and second doses between population, thus considering also the possibility that some individuals may receive only one injection if this implies the reduction of pandemic spread. By analyzing the Israeli country and its regions as a case study, this paper shows the effectiveness of the presented vaccine distribution optimal strategies.

Future research will address enhancing the model by introducing age-differentiated compartments to effectively distribute the vaccine between different classes of population. Since worldwide the healthcare systems are generally organized on a regional basis, a further development will be devoted to defining multi-region vaccine optimal distribution strategies, by allocating, for instance, more doses of the vaccine to those regions that are more affected by the pandemic or are characterized by higher infection rate.

\section{REFERENCES}

[1] P. E. Paré, C. L. Beck, and T. Başar, "Modeling, estimation, and analysis of epidemics over networks: An overview," Annu. Rev. Control, vol. 50, pp. $345-360,2020$

[2] G. Giordano, F. Blanchini, R. Bruno, P. Colaneri, et al., "Modelling the COVID-19 epidemic and implementation of population-wide interventions in Italy," Nat. Med., vol. 26, p. 855-860, 2020.

[3] C. T. Deressa and G. F. Duressa, "Modeling and optimal control analysis of transmission dynamics of COVID-19: The case of Ethiopia," Alex. Eng. J., 2020, in press, https://doi.org/10.1016/j.aej.2020.10.004.

[4] F. Della Rossa, D. Salzano, A. Di Meglio, F. De Lellis, et al., "A network model of Italy shows that intermittent regional strategies can alleviate the COVID-19 epidemic," Nat. Commun., vol. 11, p. 5106, 2020.

[5] R. Carli, G. Cavone, N. Epicoco, P. Scarabaggio, and M. Dotoli, "Model predictive control to mitigate the COVID-19 outbreak in a multi-region scenario," Annu. Rev. Control, vol. 50, pp. 373 - 393, 2020.

[6] J. Wang, Y. Peng, H. Xu, Z. Cui, and R. O. Williams, "The COVID19 vaccine race: challenges and opportunities in vaccine formulation," AAPS PharmSciTech, vol. 21, no. 6, pp. 1-12, 2020.

[7] M. Dashtbali and M. Mirzaie, "A compartmental model that predicts the effect of social distancing and vaccination on controlling COVID-19," 2020. [Online]. Available: https://europepmc.org/article/ PPR/PPR236580

[8] G. B. Libotte, F. S. Lobato, G. M. Platt, and A. J. S. Neto, "Determination of an optimal control strategy for vaccine administration in COVID-19 pandemic treatment," Computer methods and programs in biomedicine, vol. 196, p. 105664, 2020.

[9] P. Scarabaggio, R. Carli, G. Cavone, N. Epicoco, and M. Dotoli, "Nonpharmaceutical stochastic optimal control strategies to mitigate the covid-19 spread," IEEE Transactions on Automation Science and Engineering, 2021.

[10] - "Modeling, estimation, and analysis of covid-19 secondary waves: the case of the italian country," in 2021 29th Mediterranean Conference on Control and Automation (MED). IEEE, 2021, pp. 794-800.

[11] J. Grauer, H. Löwen, and B. Liebchen, "Strategic spatiotemporal vaccine distribution increases the survival rate in an infectious disease like COVID-19," Scientific reports, vol. 10, no. 1, pp. 1-10, 2020.

[12] O. Sharomi and T. Malik, "Optimal control in epidemiology," Annals of Operations Research, vol. 251, no. 1-2, pp. 55-71, 2017.

[13] B. H. Foy, B. Wahl, K. Mehta, A. Shet, G. I. Menon, and C. Britto, "Comparing COVID-19 vaccine allocation strategies in India: A mathematical modelling study," International Journal of Infectious Diseases, vol. 103, pp. 431-438, 2021.

[14] K. M. Bubar, K. Reinholt, S. M. Kissler, M. Lipsitch, S. Cobey, Y. H. Grad, and D. B. Larremore, "Model-informed COVID-19 vaccine prioritization strategies by age and serostatus," Science, vol. 371, no. 6532, pp. 916-921, 2021.

[15] E. Shim, "Optimal allocation of the limited COVID-19 vaccine supply in South Korea," Journal of clinical medicine, vol. 10, no. 4, p. 591, 2021.

[16] J. F. Robertson, H. F. Sewell, and M. Stewart, "Delayed second dose of the BNT162b2 vaccine: innovation or misguided conjecture?" The Lancet, 2021. 
[17] F. P. Polack, S. J. Thomas, N. Kitchin, J. Absalon, A. Gurtman, S. Lockhart, J. L. Perez, G. Pérez Marc, E. D. Moreira, C. Zerbini, et al., "Safety and efficacy of the BNT162b2 mRNA COVID-19 vaccine," New England Journal of Medicine, vol. 383, no. 27, pp. 2603-2615, 2020.

[18] S. Amit, G. Regev-Yochay, A. Afek, Y. Kreiss, and E. Leshem, "Early rate reductions of SARS-CoV-2 infection and COVID-19 in BNT162b2 vaccine recipients," The Lancet, 2021.

[19] "World Health Organization," https://www.who.int/emergencies/ diseases/novel-coronavirus-2019. [Accessed: 2021-06-25].

[20] "Google LLC, Google COVID-19 Community Mobility Reports," www. google.com/covid19/mobility. [Accessed: 2021-06-25].

[21] D. Welle, "Israel's clever coronavirus vaccination strategy," https://www. dw.com/en/israels-clever-coronavirus-vaccination-strategy/a-56586888 Mar 2021, [Accessed: 2021-06-25].

[22] A. W. Edridge, J. Kaczorowska, A. C. Hoste, M. Bakker, M. Klein, K. Loens, M. F. Jebbink, A. Matser, C. M. Kinsella, P. Rueda, et al., "Seasonal coronavirus protective immunity is short-lasting," Nature medicine, vol. 26, no. 11, pp. 1691-1693, 2020.

[23] P. Scarabaggio, R. Carli, G. Cavone, N. Epicoco, and M. Dotoli, "Modeling, estimation, and optimal control of anti-covid-19 multi-dose vaccine administration," in 2021 IEEE 17th International Conference on Automation Science and Engineering (CASE). IEEE, 2021, pp. 990995.

[24] "Israel's COVID-19 data dashboard," https://datadashboard.health.gov. il/\{COVID-19\}/general. Mar 2021, [Accessed: 2021-06-25]

[25] C. Santamaria, F. Sermi, S. Spyratos, S. M. Iacus, A. Annunziato, D. Tarchi, and M. Vespe, "Measuring the impact of COVID-19 confinement measures on human mobility using mobile positioning data. a european regional analysis," Safety Science, vol. 132, p. 104925, 2020.

[26] L. Guan, C. Prieur, L. Zhang, C. Prieur, D. Georges, and P. Bellemain, "Transport effect of COVID-19 pandemic in france," Annu. Rev. Control, 2020.

[27] S. M. Iacus, C. Santamaria, F. Sermi, S. Spyratos, D. Tarchi, and M. Vespe, "Human mobility and COVID-19 initial dynamics," Nonlinear Dynamics, vol. 101, no. 3, pp. 1901-1919, 2020.

[28] W.-J. Guan, Z.-Y. Ni, Y. Hu, W.-H. Liang, et al., "Clinical characteristics of coronavirus disease 2019 in China," N. Engl. J. Med., vol. 382, no. 18, pp. 1708-1720, 2020.
[29] Q. Li, X. Guan, P. Wu, X. Wang, et al., "Early transmission dynamics in wuhan, china, of novel coronavirus-infected pneumonia," N. Engl. J. Med., 2020.

[30] M. Pedersen and M. Meneghini, "Quantifying undetected COVID-19 cases and effects of containment measures in italy: Predicting phase 2 dynamics," 032020.

[31] R. Wölfel, V. M. Corman, W. Guggemos, M. Seilmaier, S. Zange, M. A. Müller, D. Niemeyer, T. C. Jones, P. Vollmar, C. Rothe, et al., "Virological assessment of hospitalized patients with COVID-19," Nature, vol. 581, no. 7809, pp. 465-469, 2020.

[32] Y. Bai, L. Yao, T. Wei, F. Tian, et al., "Presumed asymptomatic carrier transmission of COVID-19," JAMA, vol. 323, no. 14, pp. 1406-1407, 2020.

[33] Y. Liu, L.-M. Yan, L. Wan, T.-X. Xiang, et al., "Viral dynamics in mild and severe cases of COVID-19," Lancet Inf. Dis., 2020.

[34] A. L. Bertozzi, E. Franco, G. Mohler, M. B. Short, and D. Sledge, "The challenges of modeling and forecasting the spread of COVID-19," Proc. Nat. Acad. Sci., vol. 117, no. 29, pp. 16732-16738, 2020.

[35] A. P. Lemos-Paião, C. J. Silva, and D. F. Torres, "A new compartmental epidemiological model for COVID-19 with a case study of portugal," Ecol. Complex., p. 100885, 2020.

[36] M. Gatto, E. Bertuzzo, L. Mari, S. Miccoli, et al., "Spread and dynamics of the COVID-19 epidemic in italy: Effects of emergency containment measures," Proc. Nat. Acad. Sci., vol. 117, no. 19, pp. 10484-10491, 2020.

[37] K. Z. Ehmann, C. Drosten, C. Wendtner, M. Zange, et al., "Virological assessment of hospitalized cases of coronavirus disease 2019," Nature, vol. 581, pp. 465-469, 2020.

[38] F. Zhou, T. Yu, R. Du, G. Fan, et al., "Clinical course and risk factors for mortality of adult inpatients with COVID-19 in wuhan, china: a retrospective cohort study," Lancet, 2020.

[39] K. Aspelund, M. Droste, J. H. Stock, and C. D. Walker, "Identification and estimation of undetected COVID-19 cases using testing data from Iceland," NBER Work. Pap., no. w27528, 2020.

[40] MATLAB, 9.8.0.135996 (R2020b). Natick, Massachusetts: The MathWorks Inc., 2020. 